\title{
O Problema das Justificações Parciais
}

[The Problem of Partial Justifications]

\section{Alexandre Costa-Leite ${ }^{*}$}

Resumo: É comum que algumas proposições e suas respectivas negações sejam justificadas. Isso dá origem ao problema das justificações parciais. Este é apresentado e três soluções são avaliadas, discutidas e examinadas.

Palavras-chave: justificações parciais, possibilidade, contingência e indeterminação

Abstract: It is common that some propositions and their respective negations be both justified. This gives rise to the problem of partial justification. It is introduced and three solutions are evaluated, discussed and examined.

Keywords: partial justifications, possibility, contingency, indetermination

\section{1 - Introdução}

Processos justificativos, ou seja, tentativas de justificar proposições e teorias caracterizam a substância do conhecimento. Justificar significa fundamentar por via de premissas e suportes de qualquer espécie para que certas proposições possam ser aceitas como verdadeiras (ou falsas) por um agente específico ou por uma comunidade. Algumas vezes, as justificações se apresentam na forma de demonstrações e, como tais, podem ser consideradas justificações fortes e totais, uma vez que, se corretas, asseguram que a proposição justificada é, necessaria- mente, verdadeira. Outras vezes, as justificações não são suficientes para certificar a existência de uma proposição verdadeira, certa e segura. Nesse caso, elas servem apenas para estabelecer que se trata de uma proposição contingente. Tais justificações são chamadas de justificações fracas, parciais, debilitadas ou incompletas. ${ }^{1}$

Muito já foi dito e pensado sobre a noção de justificação na história da filosofia. O mesmo não pode ser afirmado sobre as lógicas da justificação. Como exemplos de investigações no contexto de sistemas formais para a justificação, temos as pesquisas e abordagens realizadas, bem recente-

\footnotetext{
${ }^{*}$ Professor adjunto do Departamento de Filosofia da Universidade de Brasília (UnB). E-mail: costaleite@unb.br. ${ }^{1}$ Cf. [4], [2] e [13].
} 
mente, por Newton da Costa em [4] e Sergei Artemov em [1]. Por um lado, a abordagem de da Costa é solitária. O autor desenvolve no livro supracitado as suas lógicas da justificação como lógicas modais. Por outro lado, o trabalho de Artemov tem atualmente atraído grande atenção por parte de pesquisadores, sobretudo, no domínio da ciência da computação. Artemov enriquece as lógicas da justificação com termos de tal modo que é possível fazer operações entre justificações. ${ }^{2}$

As duas abordagens mencionadas acima formalizam, cada uma a sua maneira, dois tipos distintos de justificação. ${ }^{3}$ As justificações fortes (totais, completas e perfeitas) são as demonstrações efetivadas no interior de sistemas formais, e elas têm algumas propriedades essenciais: ${ }^{4}$ em geral, (i) a demonstração de uma proposição implica que essa proposição é verdadeira, (ii) se uma proposição é fortemente justificada, então a sua negação não é fortemente justificada e, finalmente, (iii) se ocorre a justificação de uma proposição e a justificação de sua negação, então qualquer proposição pode ser justificada e derivada. As justificações fracas (parciais, incompletas e imperfeitas) são as mais comuns e ocorrem nas ciências, na filosofia, na política, na história, nos debates etc. Elas não possuem as propriedades (i)-(iii).

O problema avaliado neste texto pode ser formulado nas duas abordagens formais para a justificação. Concentro a atenção, entretanto, nas lógicas da justificação propostas por da Costa e mostro como o problema pode ser formulado, mutatis mutandis, para os sistemas de Artemov. Todavia, não apresento soluções para estes.

\section{2 - O problema das justificações parciais}

Considere uma proposição $\varphi$ e também a sua negação $\neg \varphi$. Lembrando que Newton da Costa utiliza $J$ em [4] para formalizar a ideia de justificação forte. Para as justificações fortes, não é possível que ocorra algo do tipo $J \varphi \wedge J \neg \varphi$ já que isso implicaria $\neg J \neg \varphi \wedge J \neg \varphi$, ou seja, uma contradição e, a partir desse ponto tudo pode ser derivado. ${ }^{5}$ A questão central deste artigo surge no contexto das lógi-

\footnotetext{
${ }^{2}$ Com a finalidade de evitar redundância na exposição, remeto o leitor ao artigo [2], o qual contém material suficiente para o entendimento deste texto. Para um estudo aprofundado sobre as diferentes lógicas da justificação, no segundo sentido, o leitor pode consultar [1].

${ }^{3}$ Detalhes em [2].

${ }^{4}$ Algumas dessas propriedades aparecem em forma axiomática no sistema de lógica da justificação proposto por da Costa em [4].

${ }^{5} \mathrm{O}$ argumento é bem simples. Detalhes podem ser encontrados em [4], p.61.
} 
cas da justificação propostas por Newton da Costa:

[...] se nosso objetivo for a edificação de uma lógica da justificação fraca, na qual $J \alpha \wedge J \neg(\alpha)$ não cause problemas de trivialização, quer levando à demonstração de qualquer fórmula do tipo $J \beta$ ou à trivialização pura e simples, deve-se enfraquecer CJ. (Newton da Costa, p.61, in [4])

Vou chamar tal questão de $o$ problema das justificações parciais (PJP): como é possível que proposições contraditórias sejam simultaneamente justificadas sem que isso destrua o sistema de inferências? Quais caminhos seguir para a construção de sistemas lógicos capazes de modelar o comportamento das justificações parciais? Avaliar, entender e apresentar três abordagens para esse problema é o interesse aqui.

\section{3 - Perspectivas e sistemas de re- ferência}

Antes de abordar (PJP), é conveniente elucidar um ponto. Uma jus- tificação total parece ser mais potente que as demais. As demonstrações são justificações fortes e, como tais, são fatuais, coerentes e não explosivas. Entretanto, surge um problema metodológico.

Considere o princípio da não contradição formulado do seguinte modo: $\neg(\varphi \wedge \neg \varphi)$. Na lógica clássica, estamos falando de uma tautologia, isto é, uma fórmula verdadeira para toda valoração. $\mathrm{E}$ isso pode ser verificado semanticamente e também por via de métodos de prova. Assim, podemos dizer que há uma justificação forte para tal princípio e, com isso, podemos afirmar que vale o princípio da não contradição. Considere agora a lógica trivalente $\mathrm{E}_{3}$ de $\mathrm{Lu}$ kasiewicz tal como definida em [10] e [6]. Usando as $Ł_{3}$-valorações é fácil ver que o princípio da não contradição obtém valor não designado para alguma valoração e, como tal, é inválido. Consequentemente, podemos também dizer que há uma justificação forte para a invalidade do princípio da não contradição. ${ }^{6}$

Diante do exposto, pode-se pensar então que há uma justificação para a validade do princípio da não contradição e que há, simultaneamente, uma justifica-

\footnotetext{
${ }^{6}$ Esse exemplo peculiar foi aqui selecionado por ser de fácil compreensão. Todavia, muitos outros argumentos semelhantes podem ser encontrados tanto na lógica quanto na matemática, como é o caso de algumas proposições da geometria euclidiana e suas rivais como as geometrias elíptica e hiperbólica.
} 
ção para a sua invalidade. Mas justificar uma proposição e justificar também a sua negação é uma característica das justificações fracas. No entanto, no caso acima, estamos diante de justificações fortes que se manifestam no interior de sistemas formais. O que está acontecendo?

A explicação é a seguinte: os sistemas de referência que são usados como parâmetros para a especificação da justificação são distintos, dado que, no primeiro caso, estamos jogando com as regras da lógica clássica e, no segundo, com as regras da lógica trivalente. Notamos, com isso, que não existem justificações totais que sejam absolutas e que justifiquem independentemente de qualquer perspectiva ou sistema de referência. Por conseguinte, uma justificação forte deve ser entendida como forte somente em relação a um dado conjunto determinado de regras. De um ponto de vista universal e geral, toda justificação pode ser vista como justificação parcial. Isso retira a hegemonia das justificações fortes. Mas é em um sistema de referência específico que uma justificação é total ou não. Por isso, para julgar uma justificação como total ou parcial é preciso saber, antes, qual o sis- tema de referência que está sendo pressuposto.

\section{4 - Justificações parciais e a pos- sibilidade lógica}

Em [2] (pág. 178), sugeri modos para a construção de sistemas de inferência capazes de modelar justificações fracas: um modo, digamos, fundado na paraconsistência e outro no tratamento da justificação fraca como um tipo de operador de possibilidade $(\diamond)$, mas não apresentei os detalhes. Faço isso agora, começando pela abordagem da justificação parcial como possibilidade. A ideia é a de que podemos pensar a justificação parcial como um tipo de possibilidade lógica (esse tipo de estratégia redutiva utilizei também no tratamento do conceito de imaginação como possibilidade lógica em [3]).

Considere um operador qualquer $*$ e as seguintes definições (estas formulam (i)-(iii) acima com mais precisão):

1. * é fatual se, e somente se, $* \varphi \rightarrow \varphi$;

2. * é coerente se, e somente se, $* \varphi \rightarrow \neg * \neg \varphi$;

3. * é explosivo ${ }^{7}$ se, e somente se, $* \varphi, * \neg \varphi \vdash \psi$.

\footnotetext{
${ }^{7} \mathrm{O}$ conceito de explosão é, em geral, usado em outro sentido na literatura (cf. [5]). Aqui a noção é definida em um sentido peculiar. Se $* \neg$ fosse equivalente a $\neg *$, teríamos o sentido comum de explosão.
} 
Para fornecer uma resposta ao (PJP), deve-se notar que um operador para modelar a justificação parcial não pode ser explosivo (no sentido acima de explosão) e não pode ser, idealmente, nem fatual e nem coerente. Em algumas situações, operadores fatuais implicam operadores coerentes.

Considere ainda a lógica modal $S 5$ e seus respectivos $\square$ e $\diamond .^{8}$ Assim, por um lado, levando em conta algumas das propriedades de $\square$ nessa lógica, podemos concluir que $\square$ de $S 5$ é fatual, uma vez que $\square \varphi \rightarrow \varphi$. Além disso, $\square$ é também coerente, pois $\square \varphi \rightarrow \neg \square \neg \varphi$ (isto é, $\square \varphi \rightarrow \diamond \varphi$ ), e é explosivo, já que $\square \varphi, \square \neg \varphi \vdash \psi$. Daí, se a base da lógica for clássica, o operador $\square$ de $S 5$ não pode ser naturalmente visto como um operador capaz de modelar a justificação fraca, embora em algumas de suas variações ele seja utilizado para pensar a justificação forte (e esse é o caminho escolhido por da Costa em [4].). Por outro lado, com o uso da semântica dos mundos possíveis, o operador $\diamond$ de $S 5$ não é fatual, dado que não vale $\diamond \varphi \rightarrow$ $\varphi$. Do mesmo modo, não há qualquer compromisso com a coerência, pois $\diamond \varphi \rightarrow \neg \diamond \neg \varphi$ não é vá- lida. Igualmente, o operador $\diamond$ não é explosivo, já que $\diamond \varphi, \diamond \neg \varphi \nvdash$ $\psi$. Como consequência, o operador $\diamond$ de $S 5$ parece ser um candidato razoável para modelar a justificação fraca.

De fato, quando estamos perante uma justificação fraca, não temos elementos suficientes para garantir que estamos lidando com uma proposição seguramente verdadeira. Se a justificação for parcial, então não há certeza sobre a verdade da proposição que é parcialmente justificada. Ela apenas pode ser verdadeira. A relação existente entre justificação parcial e possibilidade lógica parece evidente, visto que uma proposição parcialmente justificada é simplesmente possível. E a possibilidade lógica, não sendo global, exige, portanto, apenas verdade em alguma situação. Se uma proposição está fracamente justificada, então ela pode ser falsa. Esse fato está imediatamente conectado com a invalidade de $\diamond \varphi, \diamond \neg \varphi \vdash \psi$. Daí, o tratamento da justificação fraca como possibilidade lógica parece fornecer uma solução ao (PJP). ${ }^{9}$

Ainda conectado ao tema da possibilidade lógica, é interes-

\footnotetext{
${ }^{8}$ Para um estudo recente em lógica modal, o leitor pode verificar [15].

${ }^{9}$ Certamente, se a justificação fraca pode ser vista como a possibilidade em $\boldsymbol{S 5}$, então deve existir uma ligação natural entre as justificações fracas e o sistema de lógica discussiva de Jaśkowski (Cf. [8]), ou seja, conjecturo que podemos pensar a lógica discussiva como uma lógica das justificações parciais. Fundamentar essa conjectura é algo que somente poderá ser feito em outra ocasião, contudo.
} 
sante considerar quando o operador de contingência $\nabla$ é tomado como primitivo em $S 5$ (tal como desenvolvido em [11]). ${ }^{10}$ Esse operador engendra uma noção de justificação parcial. Novamente, por via da semântica dos mundos possíveis, é fácil ver que o operador $\nabla$ não é fatual já que $\nabla \varphi \rightarrow \varphi$ não é fórmula válida. Também não é coerente pois $\nabla \varphi \rightarrow \neg \nabla \neg \varphi$ não é o caso. E, por fim, $\nabla \varphi, \nabla \neg \varphi \nvdash$ $\psi$. Segue-se que é natural tratar a justificação parcial como contingência, já que esse operador satisfaz a exigência que permite uma abordagem formal ao conceito de justificação fraca.

O fato de a justificação ser parcial implica que a proposição justificada não é uma verdade necessária, dado que a justificação pode estar errada ou ser insuficiente uma vez que não justifica plenamente. Notemos, entretanto, que algumas proposições possíveis são também necessárias. Logo, pode ser mais útil caracterizar a justificação fraca como contingência, pois contingência não implica necessidade. Isso revela a dimensão vaga, incerta e imprecisa desse tipo de justificação.

\section{5 - Justificações parciais e a inde- terminação}

As justificações parciais e o respectivo (PJP) encontram uma abordagem interessante quando a justificação fraca é vista como um tipo de operador trivalente que pode ser definido no interior da lógica $Ł_{3}$ de Łukasiewicz. ${ }^{11} \mathrm{Łu}^{-}$ kasiewicz em [9] inicialmente sugere que esse valor extra capturado por $1 / 2$ seja visto como possibilidade. Leituras mais tardias mostram que tal valor pode também ser visto como indeterminação. ${ }^{12}$ Esse terceiro valor serve como base para que seja definido na linguagem de $\ell_{3}$ um operador trivalente $I$. Malinowski o interpreta como é contingente que e ainda como é modalmente indiferente. Epstein indica que tal operador é o próprio indeterminado. Tal conceito serve aqui para o objetivo de pensar a justificação fraca como o operador de indeterminação em $€_{3}$.

No ambiente trivalente de $\mathrm{E}_{3}$, são conhecidas as definições dadas para o $\diamond$ e o $\square$. A partir de tais definições, o operador $I$ é estabelecido por via da seguinte valoração: $v(\varphi)=1 / 2$ se, e somente se, $v(I \varphi)=1$. Esse operador reflete

\footnotetext{
${ }^{10}$ Propriedade básica nas lógicas da contingência é que $\nabla \varphi \leftrightarrow \nabla \neg \varphi$.

${ }^{11}$ Para um estudo sobre a hierarquia de lógicas de Łukasiewicz, o leitor pode consultar [10] e [6].

${ }^{12}$ Ver [10], pág. 18.
} 
o funcionamento interno das justificações parciais, considerando que ele não é fatual, coerente e nem explosivo. Em $Ł_{3}$, o único valor que corresponde à verdade, ou seja, o único valor que é designado é 1 . Para ver que $I$ não é fatual, ou seja, que não vale $I \varphi \rightarrow \varphi$ considere $v(\varphi)=1 / 2$. O operador $I$ também não é coerente, já que $I \varphi \rightarrow \neg I \neg \varphi$ tem valor 0 se $v(\varphi)=1 / 2$. Do mesmo modo, $I$ não é explosivo pois $I \varphi, I \neg \varphi \nvdash \psi$ quando $v(\varphi)=1 / 2$ e $v(\psi)=1 / 2$. Por conseguinte, parece razoável que a justificação parcial seja pensada e concebida como um tipo de indeterminação (e essa leitura é compatível com as anteriores que tomam justificações parciais como operadores de possibilidade e contingência, respectivamente).

Com efeito, se a justificação for fraca, então não temos razões suficientes para defender que a proposição parcialmente justificada seja completamente determinada, isto é, estamos perante uma proposição indeterminada (meramente possível, portanto). A verdade de uma proposição apenas parcialmente justificada é incerta, indeterminada. Essa leitura do terceiro valor e do operador de indeterminação no âmbito das justificações fracas pode ser vista como mais uma motivação para as lógicas polivalentes em geral. ${ }^{13}$

$\mathrm{O}$ (PJP) pode ser formulado e estudado também no horizonte das famosas lógicas da justificação ao estilo Artemov e sua escola. De fato, recentemente, ChePing Su aborda em [14] uma versão bastante parecida com o problema apresentado por da Costa em [4], mas levando em conta apenas justificações fortes. O objetivo de Su é encontrar lógicas que não satisfazem $(\square \varphi \wedge \square \neg \varphi) \rightarrow \square \psi$. Isso é o (PJP) formulado por da Costa para as justificações fortes, considerando que o operador $J$ é exatamente o operador $\square$. Mas, no contexto das lógicas de Artemov, o problema vai ser formulado com outra cara: não deve valer $\left(j: \varphi \wedge j^{\prime}: \neg \varphi\right) \rightarrow j^{\prime \prime}: \psi .^{14}$ Su então elabora uma lógica paraconsistente trivalente da justificação para responder ao problema e nessa lógica o terceiro valor utilizado é o $b$ que representa ambos verdadeiro e falso.

\footnotetext{
${ }^{13}$ Um caminho que pode ser investigado nessas relações entre polivalência e justificação é o de estabelecer níveis de justificação. Nesse sentido, (PJP) pode também encontrar uma resposta no trabalho iniciado em [2]. Outro caminho para o entendimento das justificações parciais pode ser elaborado no estilo desenvolvido em [13] quando semânticas tetravalentes são utilizadas.

${ }^{14}$ cf. [14]. Além disso, é importante notar que Artemov em [1] não faz uso daquilo que ele chama de factividade $j: \varphi \rightarrow \varphi$ e de regras de inferência para polinômios de prova. Assim, consegue pensar justificações em geral. Uma investigação completa do problema das justificações parciais no contexto de outras lógicas (e também na abordagem de Artemov) é tema fértil que pretendo desenvolver detalhadamente em outra ocasião.
} 
Há, de fato, muitas outras maneiras de resolver o problema das justificações parciais no interior da lógicas da justificação. Se se pretende manter uma leitura universal do operador ou termo de justificação, então nenhuma das vias apontadas acima é pertinente (possibilidade, contingência e indeterminação). Pelo contrário, deve-se alterar a lógica subjacente. Assim, em direção similar a de $\mathrm{Su}$, o funtor de paraconsistentização desenvolvido em [5] poderia ser aplicado às hierarquias de lógicas da justificação encontradas em [1] para uma produção de lógicas paraconsistentes da justificação. Um caminho mais simples poderia ser seguido substituindo, por exemplo, a base clássica da lógica da justificação forte de da Costa por uma lógica paraconsistente.

\section{6 - Conclusão}

Vimos qual é o problema das justificações parciais (PJP) e três maneiras de abordá-lo, todas intrinsecamente relacionadas. Mesmo sendo possível fornecer abordagens para (PJP), não há como evitar o fato de que justificações fracas, parciais, debilitadas e incompletas existem. Elas estão por todos os lados e são frequentes em domínios como a filosofia, ciências acerca da realidade e em to- dos os aspectos de nossas vidas. Tais justificações não são fatuais e nem são coerentes e, muito provavelmente, são as responsáveis por gerar teorias conflitantes e divergências de toda ordem. Nesse contexto, não há qualquer possibilidade de um conhecimento estável e claro, e não estamos em posição teórica razoável para legislar acerca do mundo em seus variados aspectos. O que temos, sim, é apenas um conjunto de afirmações parcialmente justificadas. Estamos, portanto, no âmbito das justificações de sentenças contraditórias.

Isso leva céticos ao ato de suspensão do juízo:

According to the mode deriving from dispute, we find that undecidable dissension about the matter proposed has come about both in ordinary life and among philosophers. Because of this we are not able either to choose or to rule out anything, and we end up with suspension of judgement. (Sexto Empírico, p.41, in [7])

A citação acima nos conduz até Oswaldo Porchat Pereira:

Na leitura de Sexto Empírico, encontrei a ocasião de confirmar minha experiência do conflito insupe- 
rável dos dogmatismos, de sua perpétua diaphonía. Quem longamente meditou sobre as Hipotiposes não mais ousará cometerse à edição do Discurso derradeiro. (Oswaldo Porchat Pereira, p.31, in [12])

Essa diaphonía pode ser entendida como a existência da pluralidade de justificações parciais para proposições contraditórias. Tal postura se apresenta como caminho natural se nossas teorias não são justificadas totalmente. Como o dispositivo epistêmico usado para doar verdade às proposições é vago e impreciso, como é o caso das justificações fracas, não temos elementos para saber qual é o caminho correto. Igualmente, uma vez que não há justificação total que seja absoluta, o caminho é impreciso até mesmo no âmbito das ciências formais.

Nesse sentido, do ponto de vista de agentes precários como são os agentes humanos, é curioso tentar saber se é possível a existência de uma ciência livre da pluralidade das justificações parciais.

\section{Referências}

[1] ARTEMOV, S. (2008). The logic of justification. In The Review of Symbolic Logic, 1(4), pp. 477-513.

[2] COSTA-LEITE, A. (2014). Lógicas da justificação e quase-verdade. Principia: an international journal of epistemology, 18 (2), pp.175186.

[3] COSTA-LEITE, A. (2010). Logical properties of imagination. Abstracta, 6(1), pp. 103-116.

[4] DA COSTA, N. (1999). O conhecimento científico, 2a. edição, São Paulo: Discurso Editorial.

[5] DE SOUZA, E. G.; COSTA-LEITE, A; DIAS, D.H.B. (2016). On a paraconsistentization functor in the category of consequence structures. Journal of Applied Non-Classical Logics, 26(3), pp.240-250;

[6] EPSTEIN, R. (2001). Propositional Logics: The Semantic Foundations of Logic. Belmont: Wadsworth/Thomson Learning.

[7] EMPÍRICO, S. (2005). Outlines of scepticism. Editado por Julia Annas e Jonathan Barnes. Cambridge: Cambridge University Press.

[8] JAŚKOWSKI, S. (1948). Propositional calculus for contradictory deductive systems. Logic and Logical Philosophy, 7, 35-56. 
[9] ŁUKASIEWICZ, J. (1970). On three-valued logic. In: Jan Łukasiewicz: Selected Works, editado por L. Borkowski. Warsawa: Noth-Holland Publishing. Original: 1920.

[10] MALINOWSKI, G. (1993). Many-Valued Logics. New York: Oxford University Press.

[11] MONTGOMERY, H; ROUTLEY, R. (1966). Contingency and noncontingency bases for normal modal logics. In: Logique et Analyse, vol.9, No 35-36, 318-328.

[12] PORTCHAT PEREIRA, O. (2006). Rumo ao ceticismo. São Paulo: Editora da UNESP.

[13] SCHANG, F; COSTA-LEITE, A. (2016). Une sémantique générale des croyances justifiées. CLE e-prints, 16(3), pp.1-24.

[14] SU, C-P. (2014). Paraconsistent Justification Logic: a Starting Point. Advances in Modal Logic. London: College Publications, pp. 513532.

[15] VAN BENTHEM, J. (2010). Modal logics for open minds. Stanford: CSLI Publications. 